\title{
Time-resolved 3-dimensional magnetic resonance phase contrast imaging (4D Flow MRI) analysis of hemodynamics in valve-sparing aortic root repair with an anatomically shaped sinus prosthesis
}

\author{
Thekla H. Oechtering, MD, ${ }^{a}$ Carl F. Hons, ${ }^{a}$ Malte Sieren, ${ }^{a}$ Peter Hunold, MD, ${ }^{a}$ Anja Hennemuth, PhD, ${ }^{b}$ \\ Markus Huellebrand, MSc, ${ }^{\mathrm{b}}$ Johann Drexl, MSc, ${ }^{\mathrm{b}}$ Michael Scharfschwerdt, PhD, ${ }^{\mathrm{c}}$ Doreen Richardt, MD, \\ Hans-Hinrich Sievers, MD, ${ }^{\mathrm{c}}$ Jörg Barkhausen, MD, ${ }^{\mathrm{a}}$ and Alex Frydrychowicz, $\mathrm{MD}^{\mathrm{a}}$
}

\begin{abstract}
Objective: The anatomically shaped sinus prosthesis (Uni-Graft W SINUS; Braun, Melsungen, Germany) used in valve-sparing aortic root replacement promises physiological hemodynamics believed to grant physiologic valve function. Using time-resolved 3-dimensional magnetic resonance phase contrast imaging (4D Flow MRI), we analyzed sinus vortex formation and transvalvular pressure gradients in patients with sinus prosthesis compared with age-matched and young healthy volunteers.
\end{abstract}

Methods: Twelve patients with sinus prosthesis ( $55 \pm 15$ years), 12 age-matched and 6 young healthy volunteers ( $55 \pm 6$ years, $25 \pm 3$ years, respectively) were examined at $3 \mathrm{~T}$ with a 4D flow magnetic resonance imaging sequence. Sinus vortices visualized by streamlines and time-resolved particle paths were graded on a 4-point Likert scale. Time resolved pressure differences of the left ventricular outflow tract and the ascending aorta to a reference point in the aortic bulb as well as the transvalvular pressure gradient were evaluated.

Results: 4D flow visualizations revealed a propensity of the sinus prosthesis toward intermediate $(50 \%)$ and large $(28 \%)$ vortices compared with agematched volunteers with small $(61 \%)$ and intermediate $(36 \%)$ vortices. Vortices in sinus prostheses had a similar configuration compared with those in volunteers. The peak transvalvular pressure gradient did not vary significantly between patients and age-matched volunteers $(4.0 \pm 0.9 \mathrm{~mm} \mathrm{Hg}, 3.8 \pm 0.7 \mathrm{~mm} \mathrm{Hg}$, $P=.373$ ), its temporal evolution resembled that of volunteers with a prolonged positive phase in patients.

Conclusions: Hemodynamics closely relating to those of volunteers were confirmed in sinus prostheses, believed to grant physiological valve function. Minor differences are presumably attributed to graft compliance and temporal resolution of the acquisition. Nevertheless, long-term deterioration of valve function as it was described for straight grafts could potentially be decelerated using sinus prostheses. (J Thorac Cardiovasc Surg 2016;152:418-27)

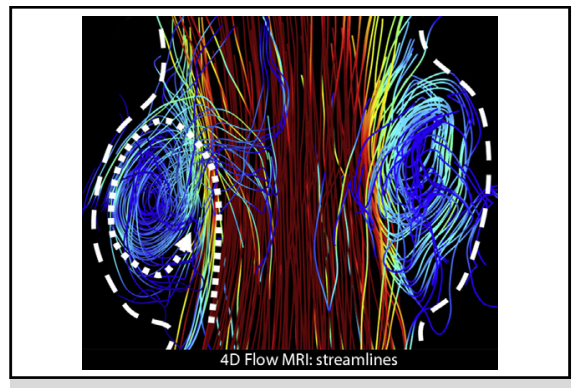

Sinus vortices in a patient with valve-sparing aortic root replacement with sinus prosthesis (male, 60 years).

\section{Central Message}

Time-resolved 3-dimensional magnetic resonance phase contrast imaging (4D Flow MRI) confirmed sinus vortex formation and transvalvular pressure gradients in patients with valve-sparing aortic root replacement with an anatomically shaped sinus prosthesis closely relating to those of agematched volunteers.

\section{Perspective}

Hemodynamics in patients with valve-sparing aortic root replacement with a sinus prosthesis closely relate to those of age-matched volunteers. This is believed to preserve native aortic valve function. Long-term deterioration of valve function as it was described for straight grafts could potentially be decelerated. Future studies are warranted to substantiate the link between postoperative hemodynamics and long-term outcome.

See Editorial Commentary page 428.
From the ${ }^{\mathrm{a}}$ Clinic for Radiology and Nuclear Medicine, and ${ }^{\mathrm{c}}$ Department of Cardiac and Thoracic Vascular Surgery, University Hospital Schleswig-Holstein, Lübeck; and ${ }^{\mathrm{b}}$ Fraunhofer MEVIS, Bremen, Germany.

Dr Anja Hennemuth, Markus Huellebrand, and Johann Drexl receive funding from Deutsche Forschungsgemeinschaft Grant ME 3330/6-1. There was no other funding provided.

Received for publication Jan 28, 2016; revisions received March 31, 2016; accepted for publication April 9, 2016.
Address for reprints: Thekla H. Oechtering, MD, Clinic for Radiology and Nuclear Medicine, University Hospital Schleswig-Holstein, Campus Lübeck, Ratzeburger Allee 160, 23562 Lübeck, Germany (E-mail: Thekla.Oechtering@uksh.de). $0022-5223 / \$ 36.00$

Copyright (C) 2016 by The American Association for Thoracic Surgery http://dx.doi.org/10.1016/j.jtcvs.2016.04.029 


\begin{tabular}{|c|c|}
\hline \multicolumn{2}{|c|}{ Abbreviations and Acronyms } \\
\hline AAO & $=$ ascending aorta \\
\hline BULB & $=$ aortic bulb \\
\hline$\Delta \mathrm{P}$ & $=$ pressure difference \\
\hline 4D Flov & $\begin{aligned} &= \text { time-resolved } 3 \text {-dimensional } \\
& \text { magnetic resonance phase contrast } \\
& \text { imaging }\end{aligned}$ \\
\hline LVOT & $=$ left ventricular outflow tract \\
\hline MRI & $=$ magnetic resonance imaging \\
\hline SP & $=$ sinus prosthesis \\
\hline SV & $=$ stroke volume \\
\hline $\mathrm{t}_{\max +/-\mathrm{n}}$ & $\begin{aligned}= & \text { time point of peak systole plus/ } \\
& \text { minus } n \text { heart phases }\end{aligned}$ \\
\hline VOL-A & $=$ age-matched volunteers \\
\hline VOL-Y & $=$ young volunteers \\
\hline VSARR & $\begin{aligned}= & \text { valve-sparing aortic root } \\
& \text { replacement }\end{aligned}$ \\
\hline
\end{tabular}

Scanning this QR code will take you to supplemental figure and videos for this article.

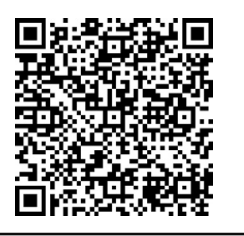

During the past 2 decades, valve-sparing aortic root replacement $(\mathrm{VSARR})^{1,2}$ has predominated alternative procedures in aortic root aneurysm repair in patients with intact aortic valve cusps. By preserving the native aortic valve, shortcomings of valve replacement such as the need for anticoagulation in mechanical valves or early degeneration of biological valves are avoided. More than the avoidance of shortcomings, however, native valve function in patients with aortic root replacement seems to be crucial.

The aortic valve forms the center of an intricate system consisting of the 3 cusps with the corresponding sinuses of Valsalva and the sinotubular junction. ${ }^{3}$ Leaflet movement is dependent strongly on the 3 sinuses. In the 16th century, Leonardo da Vinci stated that vortices in the aortic sinuses facilitate valve closure. ${ }^{4,5}$ Recent studies confirmed the importance of aortic sinuses and the vortex formation within them. They were found to contribute to proper valve opening and closure $^{3,6}$ and to minimize leaflet stress ${ }^{7}$ and transvalvular pressure gradients. ${ }^{8}$ The recirculating blood is further suspected to facilitate smooth closure of the valve, ${ }^{9}$ to promote coronary flow, and to stabilize the leaflets in open position during systole,${ }^{10}$ thereby protecting the aortic valve and potentially enhancing its longevity.

The implantation of a straight cylindrical graft as described by David and Feindel ${ }^{2}$ deprives the patient of the aortic sinuses. Frequent contact of the valve leaflets with the prosthesis wall and large bending deformation of the leaflets were observed on echocardiography and cinematography. ${ }^{11,12}$ Transvalvular pressure gradients were elevated, ${ }^{11}$ and finite element studies revealed an increased mechanical stress for the leaflets. ${ }^{7}$ Although these findings seem to have no clinical significance in the mid-term follow-up, ${ }^{13}$ they might possibly lead to preterm degenerative changes of the valve in the long term. ${ }^{3}$ Initial long-term follow-up studies suggest a slow but progressive deterioration of valve function. ${ }^{14}$

In light of these physiological blood flow properties, the introduction of the sinus prosthesis (Uni-Graft W SINUS; Braun, Melsungen, Germany) in aortic root aneurysm repair is of special interest. Its 3 separate, physiologically teardrop-formed sinuses hold the promise of preserved hemodynamic properties (Figure E1). Details on the prosthesis and the operative procedure can be found elsewhere. ${ }^{15,16}$

Time-resolved 3-dimensional magnetic resonance phase contrast imaging (4D Flow MRI) has emerged as a diagnostic technique that allows for noninvasive in vivo measurement and visualization of blood flow. Vector visualization of sinus vortices in vivo was first achieved by Kilner et al ${ }^{17}$ using 4D Flow MRI. Since then, applications have expanded from blood flow quantification, visualization, and its parametric characterization to derivation of hemodynamic and rheological properties, including the calculation of pressure differences. ${ }^{18}$

Therefore, it was the aim of this study to characterize aortic bulb hemodynamics in patients after VSARR with a sinus prosthesis (SP) by means of 4D Flow MRI. The aim of this work was 2-fold: (1) to compare the presence and extent of sinus vortices in patients with age-matched and young healthy individuals, and (2) to compare transvalvular pressure gradients between groups. In light of the physiologically shaped SP, we hypothesized that there were no differences for both vortex formation and pressure gradients.

\section{METHODS}

\section{Study Design}

Thirty-two subjects were included in this HIPAA (ie, Health Insurance Portability and Accountability Act)-compliant study after approval of the local ethics committee and written informed consent. Table 1 provides a full demographic description. Fourteen patients had undergone surgical treatment of an aortic root aneurysm with SP as described previously ${ }^{15}$ and participated in the context of routine follow-up examinations (solely root replacement, $\mathrm{n}=7$, replacement of root and ascending aorta, $\mathrm{n}=7$ ). Twelve healthy age-matched volunteers (VOL-A) and 6 young volunteers (VOL-Y) were enrolled for reference. Exclusion criteria generally were accepted contraindications against magnetic resonance imaging (MRI), failure to provide consent for the study, and arrhythmia precluding completion of 4D Flow-MR examination. Hence, 2 patients dropped out because of intermittent arrhythmia during the MRI scan preventing proper cardiac gating.

\section{MRI Scan}

A 3-dimensional, 3-directionally velocity sensitive, time-resolved phase contrast sequence was performed as part of a clinical cardiovascular 


\begin{tabular}{|c|c|c|c|c|c|c|c|c|c|}
\hline \multirow[b]{3}{*}{ Age, y } & \multirow{2}{*}{\multicolumn{2}{|c|}{ SP }} & \multirow{2}{*}{\multicolumn{2}{|c|}{ VOL-A }} & \multirow{2}{*}{\multicolumn{2}{|c|}{ VOL-Y }} & \multicolumn{3}{|c|}{$P$ value } \\
\hline & & & & & & & \multirow{2}{*}{$\begin{array}{c}\text { SP vs } \\
\text { VOL-A }\end{array}$} & \multirow{2}{*}{$\begin{array}{c}\begin{array}{c}\text { SP vs } \\
\text { VOL-Y }\end{array} \\
.002 *\end{array}$} & \multirow{2}{*}{$\begin{array}{c}\text { VOL-A vs } \\
\text { VOL-Y } \\
<.001^{*}\end{array}$} \\
\hline & $55 \pm 15$ & $(26-73)$ & $55 \pm 6$ & $(47-69)$ & $25 \pm 3$ & $(23-30)$ & & & \\
\hline Weight, $\mathrm{kg}$ & $89 \pm 9$ & $(76-100)$ & $69 \pm 13$ & $(53-92)$ & $69 \pm 10$ & $(61-85)$ & $.001 *$ & $.005 *$ & 1.000 \\
\hline Sex ratio, $\mathrm{M}: \mathrm{F}$ & $11: 1$ & & $2: 10$ & & $1: 5$ & & $<.001 *$ & $.002 *$ & 1.000 \\
\hline Height, $\mathrm{cm}$ & $183 \pm 10$ & $(160-200)$ & $171 \pm 7$ & $(161-183)$ & $173 \pm 9$ & $(163-190)$ & $.005^{*}$ & $.049 *$ & .572 \\
\hline BMI, $\mathrm{kg} / \mathrm{m}^{2}$ & $27 \pm 4$ & $(21-32)$ & $24 \pm 3$ & $(20-30)$ & $23 \pm 2$ & $(21-26)$ & $.030 *$ & $.031 *$ & .888 \\
\hline Months since surgery & $19 \pm 11$ & $(6-39)$ & - & - & - & - & - & - & - \\
\hline Bicuspid valve, $n$ & 3 & & 0 & & 0 & & .078 & .218 & 1.000 \\
\hline Marfan syndrome, $\mathrm{n}$ & 1 & & 0 & & 0 & & .359 & .556 & 1.000 \\
\hline $\begin{array}{c}\text { Antihypertensive } \\
\text { medication, } \mathrm{n}\end{array}$ & 11 & & 5 & & 0 & & $.012 *$ & $<.001 *$ & .080 \\
\hline $\begin{array}{l}\text { Blood pressure, } \\
\mathrm{mm} \mathrm{Hg}\end{array}$ & $\begin{array}{r}147 \pm 21 / \\
81 \pm 9\end{array}$ & $\begin{array}{l}(115-180 / \\
60-95)\end{array}$ & $\begin{array}{r}127 \pm 19 / \\
80 \pm 10\end{array}$ & $\begin{array}{c}(110-160 / \\
70-100)\end{array}$ & $\begin{array}{r}121 \pm 10 / \\
79 \pm 7\end{array}$ & $\begin{array}{c}(110-135 / \\
70-90)\end{array}$ & $.024 * / .501$ & $.015 * / .567$ & $.887 / .887$ \\
\hline Heart frequency, bpm & $68 \pm 11$ & $(52-87)$ & $63 \pm 9$ & $(49-76)$ & $66 \pm 6$ & $(60-72)$ & .884 & .962 & .925 \\
\hline
\end{tabular}

$S P$, Sinus prosthesis; $V O L-A$, age-matched volunteers; $V O L-Y$, young volunteers. $M$, male; $F$, female; $B M I$, body mass index. *Significant; values are given as mean \pm standard deviation (range).

MRI protocol at 3.0 Tesla (Achieva; Philips, Amsterdam, The Netherlands) with a 20-channel surface coil. The sequence was retrospectively electrocardiogram-gated. To compensate for breathing motion, respiratory gating with a 7- to 12-mm acceptance window placed on the liver-lung interface was applied to achieve a gating efficacy of approximately $65 \%$. The velocity encoding sensitivity was adapted to the expected maximal velocities and set to $180 \mathrm{~cm} / \mathrm{s}$ in all 3 spatial directions. Typical imaging parameters included isotropic voxels $\left(2.6 \times 2.6 \times 2.6 \mathrm{~mm}^{3}\right.$, interpolated to
$2.0 \times 2.0 \times 2.6 \mathrm{~mm}^{3}$ ) and a field-of-view of $380-400 \mathrm{~mm} \times 277-$ $400 \mathrm{~mm} \times 67-94 \mathrm{~mm}($ feet/head $\times$ anterior/posterior $\times$ right/left) with 25-36 slices in oblique sagittal orientation, carefully adapted to each individual's anatomy to depict the aortic arch including the aortic root. Further scan parameters were repetition time $3.6 \mathrm{~ms}$, echo time $2.3 \mathrm{~ms}$, Cartesian kspace sampling, flip angle $6^{\circ}$ in studies without contrast agent, and $12^{\circ}$ in studies with contrast agent. Contrast agent $(0.1 \mathrm{~mL} / \mathrm{kg}$ body weight, Gadobutrol, Gadovist [Bayer Vital, Leverkusen, Germany]) was administered in

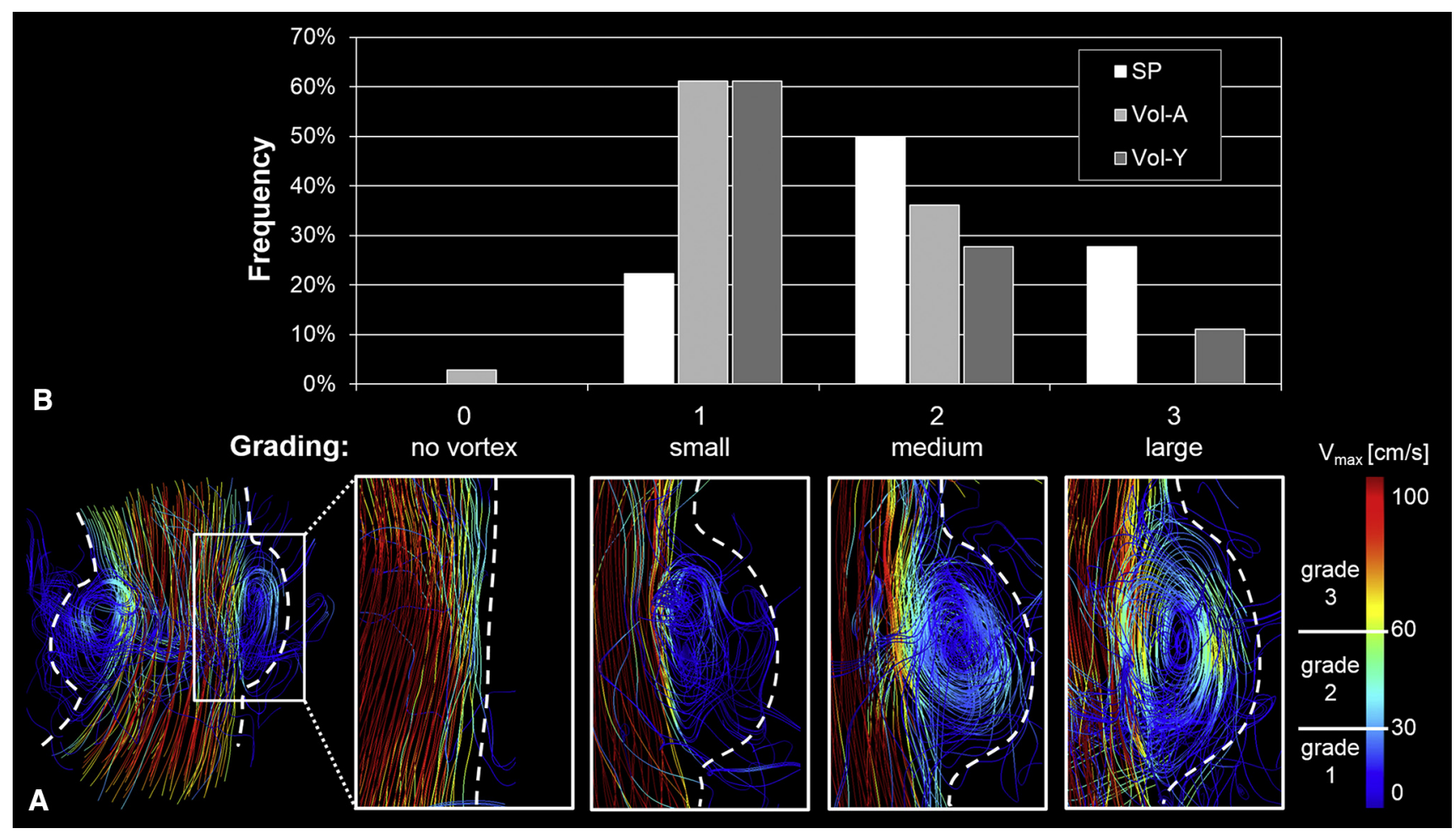

FIGURE 1. Grading of sinus vortices. A, Sinus vortices displayed as streamlines color-coded with respect to the acquired flow velocities. Vortices were graded according to their size and maximal retrograde velocity at the time point of their maximum intensity. B, The majority of sinus vortices in patients was graded medium or large whereas volunteers mostly developed small- and medium-sized vortices. SP, Sinus prosthesis; VOL-A, age-matched volunteers; $V O L-Y$, young volunteers; Vmax, peak velocity. 
12 of 12 SP and 2 of 12 VOL-A as part of a clinical protocol containing contrast-enhanced magnetic resonance angiography that was not part of this study. Image acquisition acceleration was achieved with SENSE (effective reduction factor $=2.1$ ). Data were reconstructed to 20 heart phases resulting in an effective temporal resolution of 35-62 ms, depending on heart rate. Typical scan time was $12 \pm 3$ minutes (range, 7-21 minutes), depending on respiratory and heart rate as well as navigator efficacy. As part of the clinical cardiac imaging protocol, cine short-axis balanced steady-state free precession sequences were acquired in patients and VOL-A.

\section{Processing of MRI Data}

Offline reconstruction of raw data included automated correction of eddy currents and Maxwell terms. Data were transferred to an onsite computer equipped with a commercially available software GTFlow (v2.1.4; GyroTools LLC, Zurich, Switzerland) used for visualization and the scientific software MEVISFlow (v9.1b; Fraunhofer MEVIS, Bremen, Germany) used for semiautomated pressure difference estimation. ${ }^{19}$ In 5 patients, data processing included aliasing correction with PhaseUnwrappingTool (v1; Fraunhofer MEVIS).

For blood flow visualization, a 2-dimensional contour transecting the aortic bulb in a fashion that all sinuses could be differentiated was carefully placed in GTFlow. From this contour, hemodynamic parameters such as stroke volume (SV), peak velocity, and flow volumes were derived. To visualize hemodynamics, particle paths and instantaneous streamlines, colorcoded to the acquired flow velocity, were emitted in 3-dimensional mode from the predefined contour. Streamlines and time-resolved particle paths passing the contour during the heart cycle were visualized downstream to the contour as well, so-called forward and backward tracing, so that small flow patterns that developed directly distal or at the same level as the contour were visualized. Further details on visualization approaches can be found elsewhere. ${ }^{20-22}$

For sinus vortex analysis, streamlines and time-resolved particle paths were used. Sinus vortices form in the aortic sinuses behind the opened valve cusps and are defined as recirculating blood deviating from main flow direction. ${ }^{23}$ Each vortex in the left, right, and noncoronary sinus was graded visually according to its size and maximal retrograde velocity at the time point of its maximal intensity on a 0-3 scale, with " 0 " indicating no vortex was detected. A maximal retrograde velocity of $30 \mathrm{~cm} / \mathrm{s}$ or $60 \mathrm{~cm} / \mathrm{s}$ was considered a small or intermediate vortex, and greater retrograde velocities than $60 \mathrm{~cm} / \mathrm{s}$ indicated a large vortex (Figure 1, $A$ ). To describe the temporal evolution of sinus vortex formation, the number of heart phases between peak systole and maximal vortex intensity was noted as well as a persistence into diastole.

Pressure difference $(\Delta \mathrm{P})$ calculation was achieved with MEVISFlow after semiautomatically segmenting aorta and left ventricular outflow tract (LVOT; Figure 2, A). First, a relative pressure map was calculated solving the Navier-Stokes equation based on the acquired time-resolved velocity fields assuming blood as a viscous, incompressible fluid. Viscosity was set at $0.0045 \mathrm{~Pa} \cdot \mathrm{s}$ and density at $1055 \mathrm{~kg} / \mathrm{m}^{3}$. A reference point (aortic bulb $[B U L B])$ was positioned at the level of the vena contracta, the location of the narrowest flow jet downstream of the aortic anulus, where pressure at peak systole $\left(\mathrm{t}_{\max }\right.$, defined as the time point of peak velocity in the aortic bulb) dropped to a minimum. The reference value in the aortic bulb was set to $0 \mathrm{~mm} \mathrm{Hg}$. To calculate $\Delta \mathrm{P}, 2$ contours were positioned $3 \mathrm{~cm}$ upstream in the LVOT and $4 \mathrm{~cm}$ downstream the aortic valve in the ascending aorta (AAO). $\Delta \mathrm{P}$ of the contours relative to the reference point was measured in every phase of the cardiac cycle. The transvalvular pressure gradient $\Delta \mathrm{P}($ LVOT,AAO) was calculated by subtracting $\Delta \mathrm{P}(\mathrm{AAO})$ from $\triangle \mathrm{P}($ LVOT). Its maximum value, the peak transvalvular pressure gradient, was noted in every subject.

Ejection fraction and SV were determined from short-axis SSFP cine sequences following clinical standard by use of the Extended MR WorkSpace (v2.6.3.5; Philips). Healthy young volunteers without a history of cardiovascular disease were defined to have a normal ejection fraction.

\section{Statistics}

Results with continuous values are given as mean \pm standard deviation (range). Results of sinus vortex grading and time of maximal vortex intensity are given as median $[25 \%, 75 \%$ percentile $]$. For comparison between groups, a Mann-Whitney $U$ test was performed. A value of $P<.05$ was considered statistically significant. Correction for multiple testing was not performed because equality was to be proven; hence, second-order error was of greater concern than first order error.

\section{RESULTS \\ Quantification and Visualization of Hemodynamics}

Patients with SP and VOL-A had a normal ejection fraction, although patients with SP had a lower ejection fraction than VOL-A $(P=.022)$. SV, heart rate, and cardiac index did not differ significantly between SP and VOL-A (Table 2).

Sinus vortices were confirmed in the sinuses of Valsalva of all subjects (Figure 3, Video 1, $A-C$ ) except for a noncoronary sinus of 1 VOL-A. In patients, sinus vortices were predominantly intermediate or large (median grading: 2, [2, 3]) compared with small- and intermediate-sized vortices in VOL-Y and VOL-A (median grading: 1 [1, 2] for both groups; for all, $P<.05$; Figure 1 , $B)$. There was no significant difference between VOL-Y and VOL-A $(P=.56)$. Vortex intensity in the left, right, and noncoronary sinus did not differ significantly (for all, $P>.05)$. Time of maximal sinus vortex intensity was $0[0,1]$ heart phases after peak systole in SP compared with $1[0,1]$ and $1[0.75,1.25]$ in VOL-A and VOL-Y, respectively, and did not differ significantly between SP and VOL-A $(P=.3)$. All sinus vortices persisted into early diastole.

\section{Pressure Differences}

With 4D Flow MRI, the temporal evolution of pressure gradients across the aortic valve was analyzed successfully in all subjects. In 2 patients, the pressure minimum at peak systole was not detected in the aortic bulb but in the ascending aorta, where flow velocities were greater than in the vena contracta (Figure 4). These 2 patients were excluded from statistical analysis. Overall, physiological changes could be confirmed in all groups as described for each location in the following paragraphs.

Peak pressure gradients across the aortic valve $\triangle \mathrm{P}(\mathrm{LVOT}, \mathrm{AAO})$ in early systole were $4.0 \pm 0.9 \mathrm{~mm}$ $\mathrm{Hg}$ in patients compared with $3.8 \pm 0.7 \mathrm{~mm} \mathrm{Hg}$ in volunteers (VOL-A, $3.5 \pm 0.6 \mathrm{~mm} \mathrm{Hg}$; VOL-Y, $4.3 \pm 0.6 \mathrm{~mm}$ $\mathrm{Hg}$ ). The difference between patients and volunteers did not prove significant. Differences between VOL-Y and VOL-A revealed statistical significance $(P=.028$; Figure 5). 

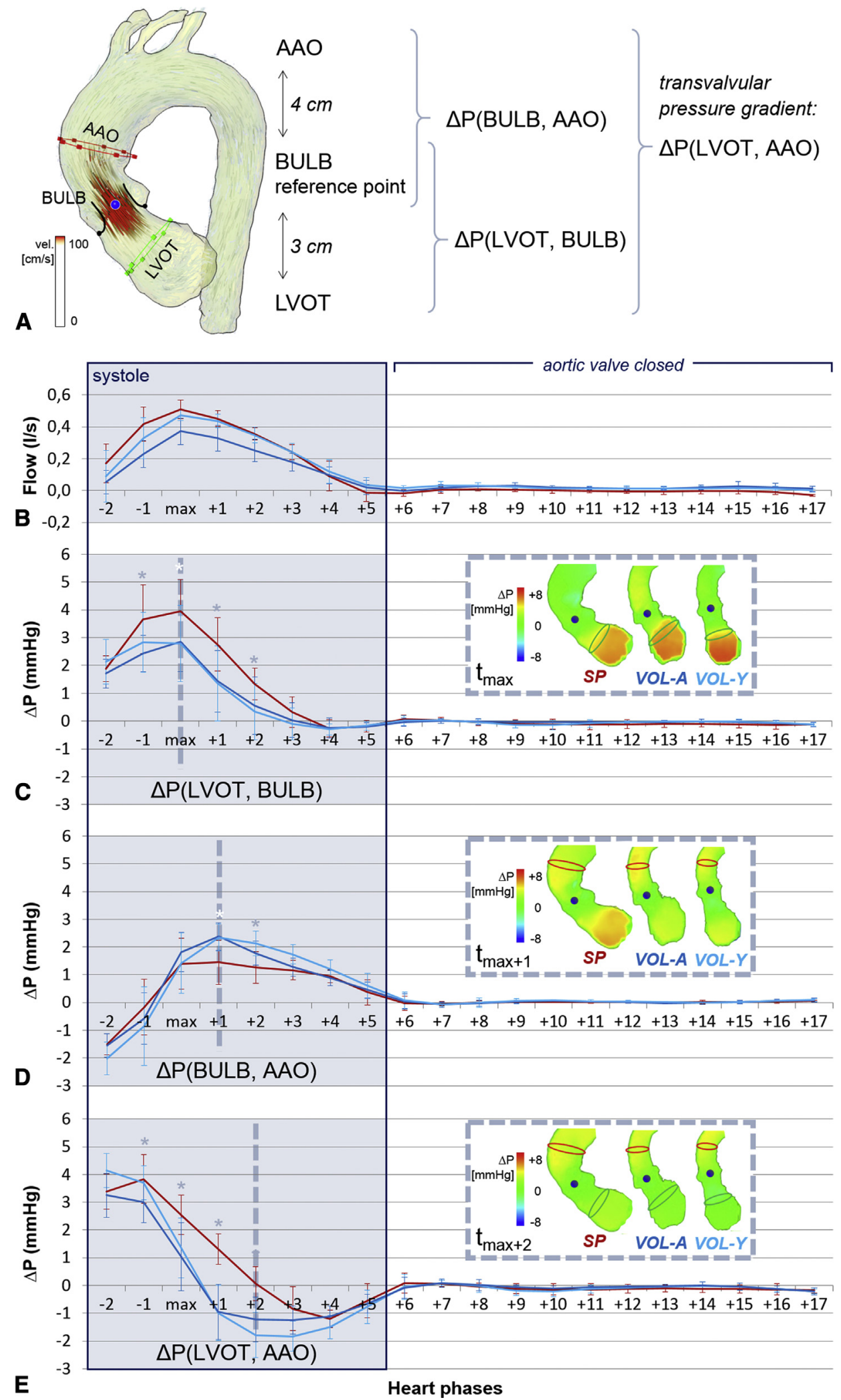

FIGURE 2. Relative pressure differences. A, A reference point (blue circle) for $\Delta \mathrm{P}$ calculation was positioned at the level of maximum systolic flow velocity, where the diameter of the flow jet reaches its minimum in the aortic bulb (BULB). Two contours were positioned in the left ventricular outflow tract (LVOT, green) and the ascending aorta (AAO, red). Three pressure gradients were derived: $\triangle \mathrm{P}(\mathrm{LVOT}, \mathrm{BULB}), \Delta \mathrm{P}(\mathrm{BULB}, \mathrm{AAO})$, and the transvalvular 
The transvalvular pressure gradient was positive while flow velocity increased in early systole. It decreased rapidly until it became negative just after peak systole, when velocity started to decrease as well. Fundamentally, aortic pressure exceeded ventricular pressure in late systole while blood was still being ejected from the ventricle. With 4D Flow MRI, the evolution of transvalvular $\Delta \mathrm{P}$ [ $\triangle \mathrm{P}(\mathrm{LVOT}, \mathrm{AAO})]$ was ascertained in all volunteers. Pressure gradient development in patients resembled that of volunteers except for a deceleration of the decrease of transvalvular $\Delta \mathrm{P}$ in patients (Figure 2,E). This results in a longer phase of positive relative pressure in the LVOT and a shorter phase of positive relative pressure in the AAO. Considering a delay of 1 heart phase in patients for the first part of systole, there was no significant difference between patients and volunteers except for a greater $\Delta \mathrm{P}$ at the time frame after peak systole in patients (Figure 2, E).

Relative pressure in the LVOT was increased relative to the bulb in early systole, promoting blood ejection into the aorta. $\triangle \mathrm{P}(\mathrm{LVOT}, \mathrm{BULB})$ peaked at maximum blood flow velocity (Figure $2, C$ ). Subsequently $\Delta \mathrm{P}(\mathrm{LVOT}, \mathrm{BULB})$ decreased rapidly until leveling out with relative bulb pressure in diastole. Patients revealed an increased $\Delta \mathrm{P}$ (LVOT,BULB) (SP vs VOL-A, $P<.05$ from $\mathrm{t}_{\max -1}$ to

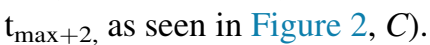

$\triangle \mathrm{P}(\mathrm{BULB}, \mathrm{AAO})$ initially was negative but rapidly exceeded the pressure of the bulb between $t_{\max -1}$ and $t_{\max }$, reaching its maximum after peak systole, then plateauing until slowly decreasing until the end of systole (Figure 2, $D$ ). After peak systole, patients had a less-pronounced pressure increase than volunteers with a significant difference at $\mathrm{t}_{\max +1}$ and $\mathrm{t}_{\max +2}$ between SP and VOL-A $(P=.007$ and .010 , respectively).

\section{DISCUSSION}

This study carries 2 messages we believe to be important. First, anatomically shaped aortic root prostheses exhibited hemodynamics that closely relate to those of age-matched volunteers with a tendency towards more pronounced sinus vortex formation. Second, transvalvular pressure gradients were of comparable amplitude but revealed a slight temporal delay. The results substantiate the high potential of $4 \mathrm{D}$ Flow MRI to evaluate postoperative hemodynamics in the aorta simultaneously with confirming the hypothesized physiological hemodynamics of an anatomically shaped aortic root prosthesis.
Sinus vortices persisted into early diastole, consistent with previous works. ${ }^{5,24}$ Sinus vortex formation is congruent with previous ultrasound studies confirming that valve leaflets do no collide with the wall of the SP during systole. ${ }^{15,16}$ In detail, vortices developed in early systole with maximum intensity about 1 heart phase after peak systole, concordant between groups. While in general there were larger vortices in patients, the per-sinus analysis across all participants did not show differences in vortex strength between sinuses. This result differs from those of both Kvitting et al, ${ }^{24}$ who found that vortices developed only after peak systolic ejection velocity with relatively larger vortices in right and noncoronary sinuses, and Markl et al, ${ }^{25}$ who described more pronounced vortices in the left and right coronary sinus. This difference might be explained by smaller volunteer collectives, differing scan parameters, and foremost by different analysis strategies. Frequently, streamlines and particle paths are emitted from a particular contour only. In contrast, our analysis additionally included streamlines and particle paths starting upstream to the contour and passing through it; therefore, a denser and probably more reliable visualization can be achieved. ${ }^{26}$ The comparison of visualization strategies, however, warrants further investigation.

Although the clinical relevance of sinus vortex size is not yet clear, one can state that too small vortices bear the risk of collision of the valve leaflets with the vessel wall, whereas too large vortices bear the risk of energy dissipation, which may lead to an increased strain of the left ventricle. Vortices in SP patients were larger than those in volunteers. There were no significant differences in aortic bulb diameters between patients and VOL-A that were able to justify the increased vortex formation. We hypothesize that, next to differences in cardiac output, the reduced compliance $^{16}$ and Windkessel effect of the prosthesis not only contributes to increased bending deformation of the valve leaflets ${ }^{16}$ but induces an augmented resistance and backward flow and thus increased vortices.

In addition, the administration of contrast agent in patients may have prompted an increased signal and thus better visualization quality. To at least partially compensate for such effects, the flip angles were adapted to the absence of contrast agents. Further mitigating the potential influence of contrast agent are data in 2 volunteers with a sinus vortex intensity comparable to the other volunteers who did not receive contrast agent. Because the retrograde velocity of sinus vortices is dependent on the flow velocity

pressure gradient $\triangle \mathrm{P}(\mathrm{LVOT}, \mathrm{AAO})$. B, Flow profile in the aortic bulb over the course of time (20 heart phases: $\mathrm{t}_{\max -2}$ to $\mathrm{t}_{\mathrm{max}+17}$ with $\mathrm{t}_{\mathrm{max}}$ as peak systole). Pressure differences $(\triangle \mathrm{P})$ between $\mathrm{C}$, LVOT or D, AAO and BULB as well as E, the transvalvular pressure gradient $\Delta \mathrm{P}(\mathrm{LVOT}$, AAO) in patients with sinus prosthesis (SP: red) and age-matched and young volunteers (VOL-A: dark blue and VOL-Y: light blue, respectively) reveal physiological behavior. The transvalvular pressure gradient is positive in early systole and gets negative after peak systole while blood is still being ejected from the ventricle. Examples for each group at defined time points in the boxes on the right. [*SP vs VOL-A: $P<.05$ ]. AAO, Ascending aorta; $B U L B$, aortic bulb; $L V O T$, left ventricular outflow tract; $\triangle P$, pressure difference; tmax $+/-n$, time point of maximum systole plus/minus $\mathrm{n}$ heart phases; $S P$, sinus prosthesis; $V O L-A$, age-matched volunteers; VOL-Y, young volunteers. 
TABLE 2. Hemodynamic parameters and bulb diameter

\begin{tabular}{|c|c|c|c|c|c|c|}
\hline & \multirow[b]{2}{*}{ SP } & \multirow[b]{2}{*}{ VOL-A } & \multirow[b]{2}{*}{ VOL-Y } & \multicolumn{3}{|c|}{$P$ value } \\
\hline & & & & SP vs VOL-A & SP vs VOL-Y & VOL-A vs VOL-Y \\
\hline \multicolumn{7}{|l|}{ Diameter } \\
\hline Bulb, cm & $\begin{array}{c}3.6 \pm 0.2 \\
\text { (range, } 3.3-3.9 \text { ) }\end{array}$ & $\begin{array}{c}3.3 \pm 0.4 \\
\text { (range, } 2.8-4.1 \text { ) }\end{array}$ & $\begin{array}{c}3.3 \pm 0.1 \\
\text { (range, } 3.1-3.4 \text { ) }\end{array}$ & .105 & $.010^{*}$ & .925 \\
\hline \multicolumn{7}{|l|}{ Heart function } \\
\hline $\mathrm{EF}, \%$ & $58.4 \pm 7.4$ & $65.4 \pm 2.8$ & - & $.022 *$ & - & - \\
\hline Stroke volume, $\mathrm{mL}$ & $100.6 \pm 23.7$ & $81.7 \pm 10.5$ & - & .069 & - & - \\
\hline Cardiac output, L/min & $6.6 \pm 0.9$ & $5.1 \pm 1.0$ & - & $.002 *$ & - & - \\
\hline Cardiac index, $(\mathrm{L} / \mathrm{min}) / \mathrm{m}^{2}$ & $3.1 \pm 0.4$ & $2.8 \pm 0.4$ & - & .093 & - & - \\
\hline \multicolumn{7}{|l|}{ Hemodynamic parameters } \\
\hline Stroke volume, $\mathrm{mL}$ & $102 \pm 22$ & $91 \pm 19$ & $111 \pm 10$ & .225 & .206 & $.019 *$ \\
\hline Peak average velocity, $\mathrm{mL}$ & $59 \pm 8$ & $50 \pm 7$ & $62 \pm 6$ & $.009 *$ & .280 & $.006^{*}$ \\
\hline Peak maximum velocity, $\mathrm{cm} / \mathrm{s}$ & $177 \pm 54$ & $130 \pm 15$ & $136 \pm 13$ & $.002 *$ & $.031 *$ & .542 \\
\hline Peak net flow, $\mathrm{mL} / \mathrm{s}$ & $512 \pm 78$ & $372 \pm 78$ & $477 \pm 41$ & $<.001 *$ & .425 & $.022 *$ \\
\hline
\end{tabular}

$S P$, Sinus prosthesis; $V O L$-A, age-matched volunteers; $V O L-Y$, young volunteers; $E F$, ejection fraction; $B M I$, body mass index. *Significant; values are given as mean \pm standard deviation.

in the center of the aortic bulb, ${ }^{27}$ greater maximal flow velocities in the bulb of SP patients may contribute to larger sinus vortices.

Similarly, 4D Flow MRI was able to characterize the not entirely intuitive temporal evolution of transvalvular $\Delta \mathrm{P}$, consistent with results from invasive measurements ${ }^{28,29}$ : ventricular pressure exceeds aortic pressure only in the first part of systole, promoting left ventricular outflow into the aorta. Transvalvular $\Delta \mathrm{P}$ rapidly decreases until aortic pressure exceeds ventricular pressure in the second part, just after peak systole, while blood is still being ejected from the ventricle into the aorta.

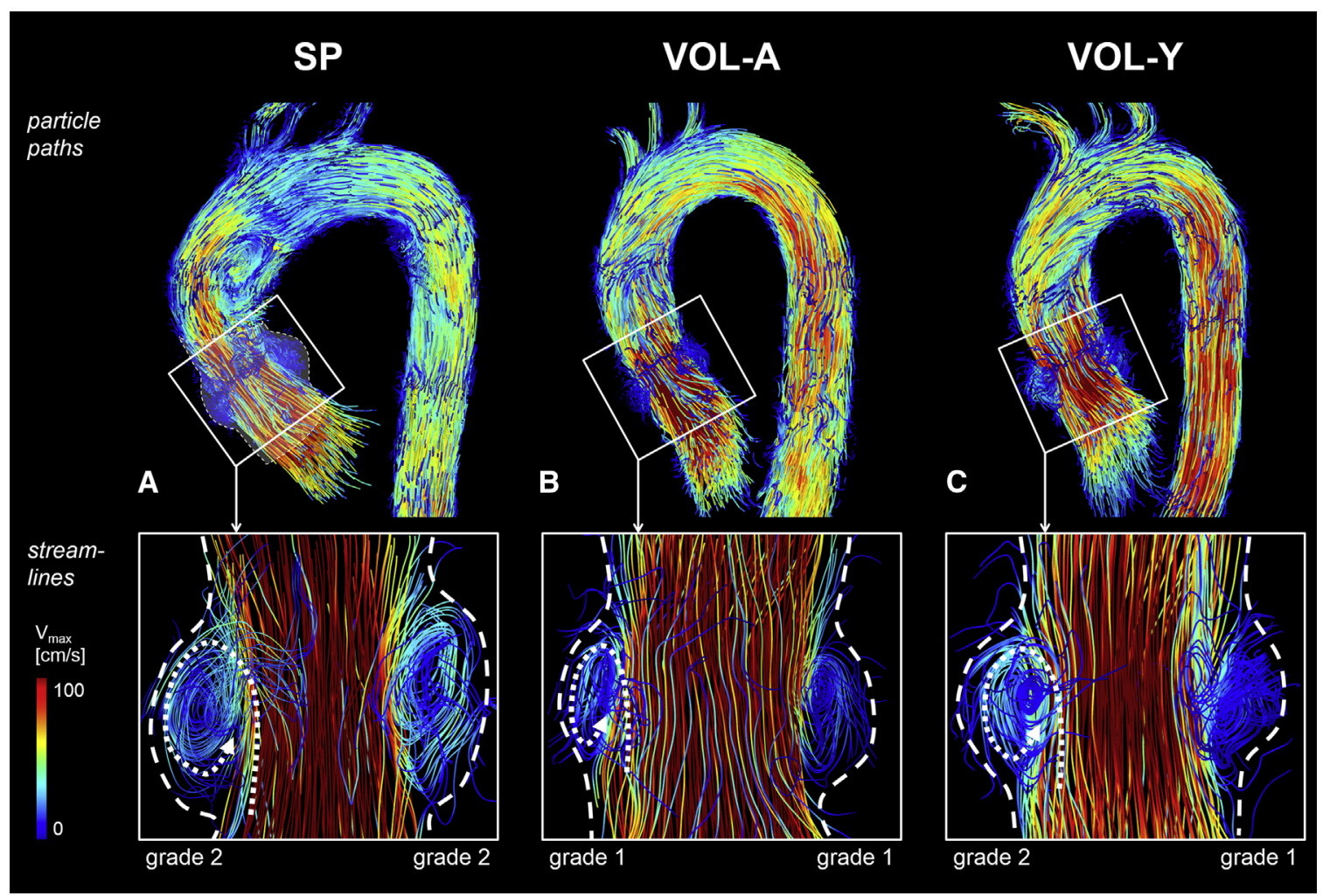

FIGURE 3. Sinus vortices. Top row: particle paths at peak systole delineate hemodynamics in the thoracic aorta of 60-year-old patient with SP (A), of a healthy 53-year-old VOL-A (B), and a 30-year-old VOL-Y (C). Bottom row: sinus vortices in the right and left coronary sinus as visualized by instantaneous streamlines. Sinus vortices develop behind the opened valve cusp in each coronary sinus during systole and persist until early diastole. Vortices in patients had a similar configuration than those in volunteers. Dashed lines are used to emphasize sinus borders and dotted lines to underline vortex direction. SP, Sinus prosthesis; VOL-A, age-matched volunteers; VOL-Y, young volunteers; Vmax, peak velocity. 

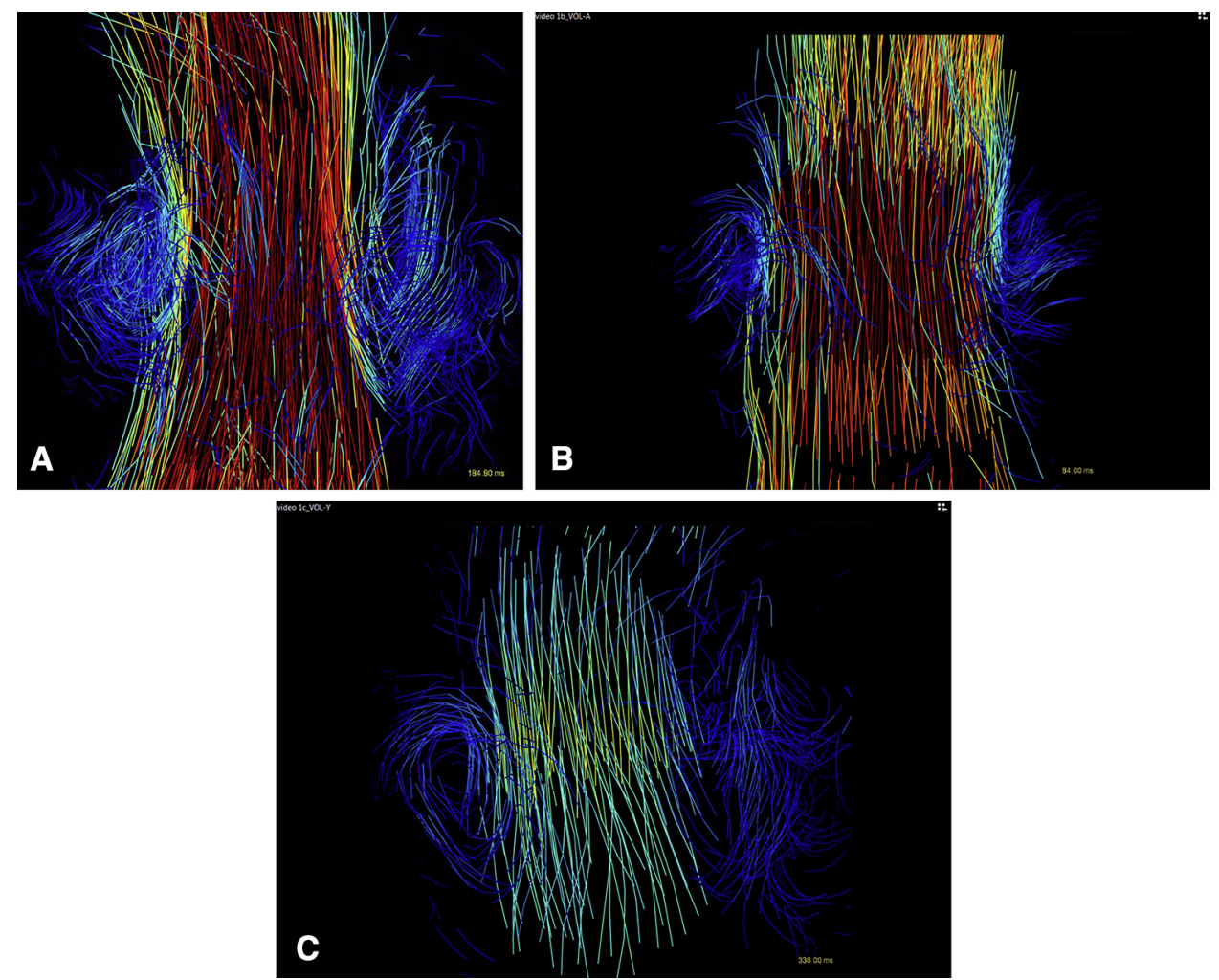

VIDEO 1. Sinus vortices. Particle paths of sinus vortices in A, a 60-year-old patient with sinus prosthesis; B, a healthy 53-year-old age-matched patient; and C, a 30-year-old young volunteer. Sinus vortices develop behind the opened valve cusp in each coronary sinus during systole and persist until early diastole. Vortices in patients had a similar configuration than those in volunteers.
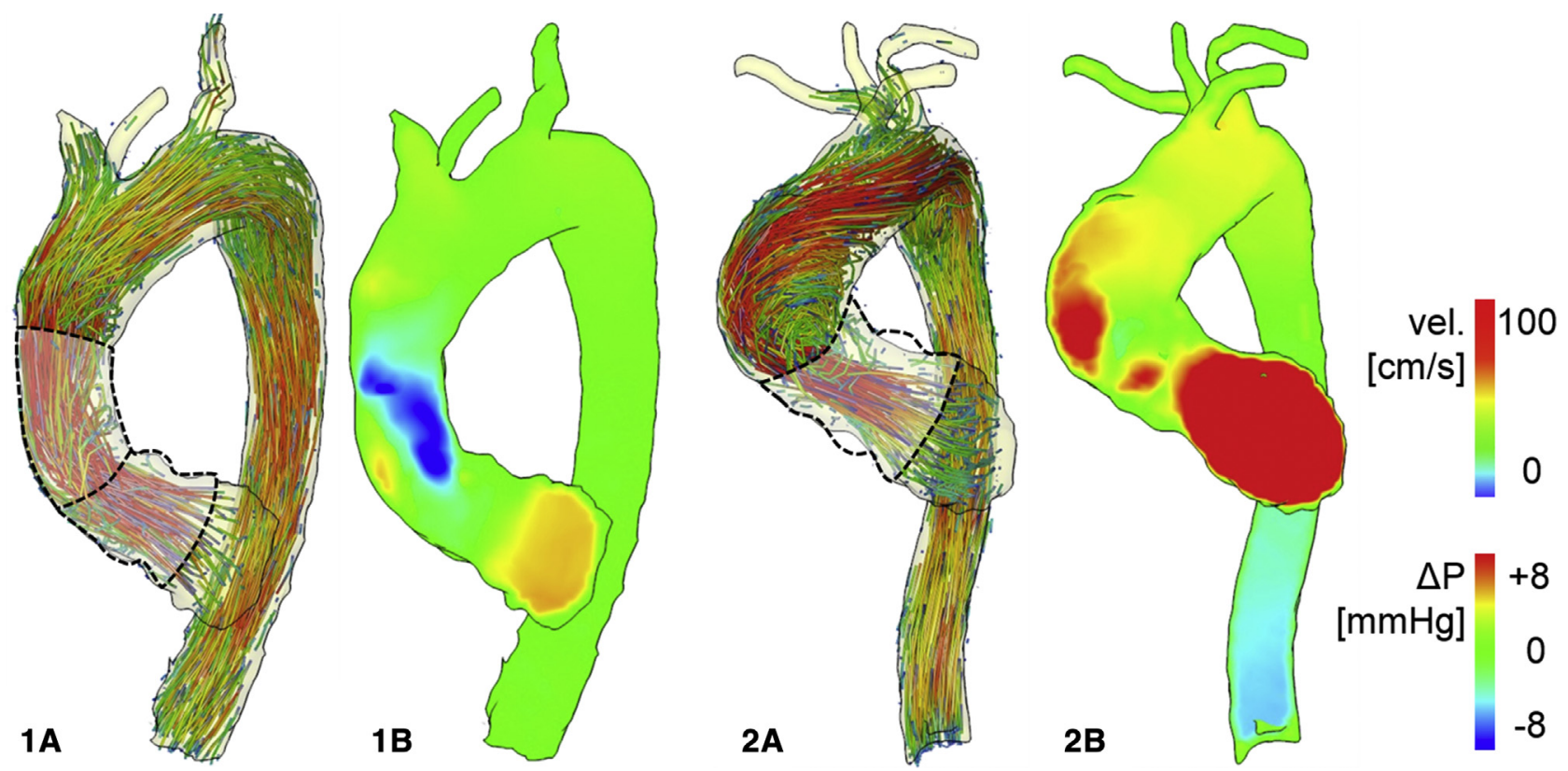

FIGURE 4. Patients with pressure drops in the ascending aorta. In 2 patients, the pressure minimum was not detected in the bulb but in the ascending aorta. In the first (male, 57 years) a secondary helix developed distal to a kinking of the prosthesis (1A) resulting in a pronounced pressure drop in the ascending aorta (1B). The second patient (female, 61 years) had a stenotic bicuspid aortic valve causing a vortex in the ascending aorta (2A) with an accompanying pressure drop (2B). Prostheses were delineated retrospectively as dotted lines. A, particle paths; B, pressure map. $\Delta P$, Pressure difference. 


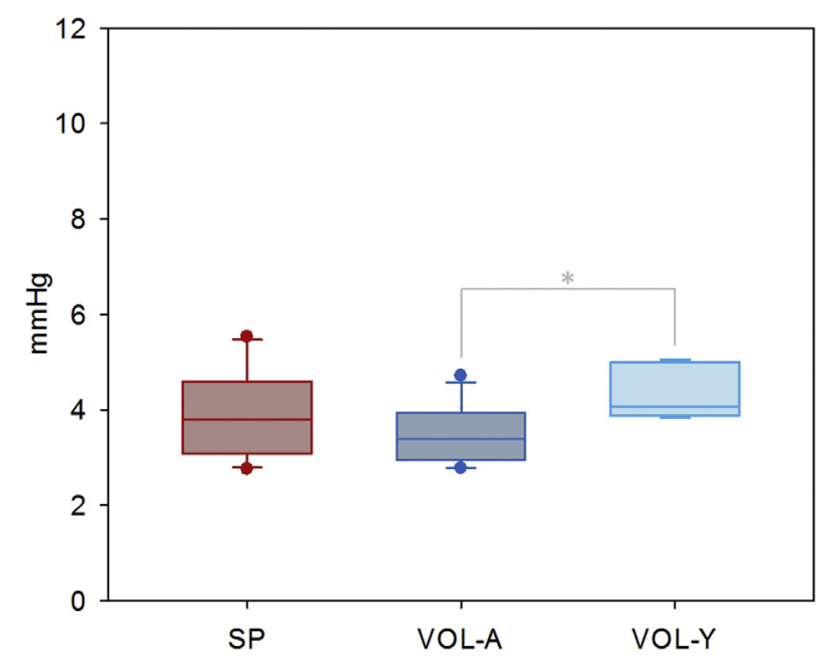

FIGURE 5. Peak transvalvular pressure gradients. Peak transvalvular pressure gradients did not differ significantly between patients with SP and volunteers. In contrast, differences between VOL-Y and VOL-A were statistically significant. $S P$, Sinus prosthesis; VOL-A, age-matched volunteers; VOL-Y, young volunteers. *Statistical significance, $P<.05$.

A pressure gradient in an elastic pipe is almost proportional to the acceleration or deceleration of a fluid. ${ }^{28}$ The ascending aorta with its Windkessel effect is such an elastic pipe. Positive transvalvular $\Delta \mathrm{P}$ triggers the steep upslope of the velocity curve in the first part of systole. The more gradual decrease of blood flow velocity in the second part of systole is a result of negative transvalvular $\Delta \mathrm{P}$. It allows for a gradual initiation of valve closure and thus contributes to physiological valve function. ${ }^{30}$

SP demonstrated a prolonged positive transvalvular pressure gradient as a result of an increased LVOT pressure and a significantly reduced aortic pressure. In SP there was neither a reduction of aortic blood flow nor an elevation of transvalvular $\Delta \mathrm{P}$. The prolonged positive transvalvular $\Delta \mathrm{P}$ may be attributed to the reduced compliance of the synthetic prosthesis and hence increased resistance compared with the natural aortic bulb. In noncompliant vessels, pressure waves travel faster, thus getting reflected earlier, and consequently leading to a systolic pressure increase in the ascending aorta. Secondary flow patterns in the ascending aorta could influence aortic pressure as well; however, this was not within the scope of this study and will have to be addressed in following investigations.

Peak transvalvular pressure gradients did not differ significantly between volunteers and patients further underlining the close correlation of hemodynamics in sinus prostheses and volunteers. Findings are consistent with echocardiographic measurements ${ }^{15,16}$; however, the absolute level of 4D Flow MRI-derived pressure differences (4.0 \pm $0.9 \mathrm{~mm} \mathrm{Hg}$ ) is markedly lower than those described in previous in vivo studies with echocardiography (8.1 \pm $3.6 \mathrm{~mm} \mathrm{Hg})^{15}$ and invasive measurements in phantoms (8.9 $\pm 1.1 \mathrm{~mm} \mathrm{Hg}) .{ }^{16}$ We attribute this difference to the fact that pressure gradients were derived from the velocity field of the entire vessel cross section in contrast to the peak velocity in the center of the vessel. 4D Flow MRI has been demonstrated previously to be both reliable and accurate. ${ }^{18,31,32}$ In general, it shows a tendency to underestimate peak flows and pressure gradients, presumably explained by spatiotemporal averaging during MRI acquisitions. ${ }^{18}$

As part of the $\Delta \mathrm{P}$ characterization, both the Venturi effect and Bernoulli's principle, the first describing a reduction of pressure when a fluid passes a constricted section of a pipe, the second proposing an increase in velocity after the decrease of pressure, was affirmed. At peak systole, increased relative pressures in LVOT and AAO compared with the center of the aortic bulb were verified. Deducting that valve closure is promoted by the Venturi effect would profit from an analysis of pressure differences across a vessel cross section, which was limited by the acquired spatial resolution.

In 2 patients, the pressure minimum at peak systole was not detected in the aortic bulb but was displaced further downstream in the ascending aorta (Figure 4). In the first patient, both the aortic bulb and ascending aorta were replaced. In consequence, a kinking between both grafts had developed, promoting a secondary helix within the prosthesis. Similarly, the second patient presented with a stenotic bicuspid aortic valve and a postprosthetic dilatation, which resulted in a large ascending aortic vortex. We consider the aforementioned secondary flow patterns responsible for a restricted forward flow to the respective small part of the vessel lumen resulting in a displaced pressure minimum.

Shortcomings of this work are the relatively small collective of patients and long acquisition and postprocessing times. As of now, there is a limited number of patients with SP from which to recruit. Therefore, the presented results have to be understood as promising pilot data on which to expand a clinical study in which the connection between hemodynamics and long-term outcome not only in SPs but also in other VSARR prostheses needs to be analyzed. This will also help to discriminate flow patterns induced by the prosthesis or surgery itself. Moreover, potential characteristic features of patients with connective tissue disorders need to be investigated.

First mid-term-outcomes of a Valsalva graft with pseudosinus are promising. ${ }^{33}$ The relatively long acquisition times that may lead to patient discomfort on one hand and averaging of small cyclic flow patterns on the other hand will be overcome by optimized parallel data acquisition techniques. ${ }^{34,35}$ At the time of the study, however, these techniques were not available. Lastly, long postprocessing may limit clinical practicability. Although modern imaging centers provide postprocessing services, the applied software is of scientific nature and not approved by the Food and Drug Administration and postprocessing is time-consuming, which hampers its clinical applicability. As 4D Flow MRI increasingly contributes to clinical research, however, this missing step may be overcome in future as well. 


\section{CONCLUSIONS}

Although flow patterns within the sinuses and pressure gradients were both confirmed to be closely related to those of age-matched volunteers (VOL-A), future studies are necessary to clarify whether sinus prostheses have an impact on the long-term outcome of patients compared with straight grafts. Sinus vortex formation was comparable with those of volunteers believed to enable physiological valve function and thus decreased stress on the valve leaflets. Together with physiological pressure gradients, this could possibly lead to less valve deterioration in the long term, which seems especially relevant in particular for younger patients, eg, with Marfan syndrome.

\section{Conflict of Interest Statement}

Prof. Sievers receives royalties from B. Braun Melsungen AG. All other authors have nothing to disclose with regard to commercial support.

The authors express their gratitude toward Mrs. Martina Schröder for her skillful assistance and Dr Gérard Crelier for his continuous support.

\section{References}

1. Sarsam MA, Yacoub M. Remodeling of the aortic valve anulus. J Thorac Cardiovasc Surg. 1993; 105:435-8.

2. David TE, Feindel CM. An aortic valve-sparing operation for patients with aortic incompetence and aneurysm of the ascending aorta. J Thorac Cardiovasc Surg. 1992; 103:617-22.

3. Robicsek F, Thubrikar MJ, Fokin AA. Cause of degenerative disease of the trileaflet aortic valve: review of subject and presentation of a new theory. Ann Thorac Surg. 2002;73:1346-54.

4. Robicsek F. Leonardo da Vinci and the sinuses of Valsalva. Ann Thorac Surg. 1991;52:328-35.

5. Bissell MM, Dall'Armellina E, Choudhury RP. Flow vortices in the aortic root: in vivo 4D-MRI confirms predictions of Leonardo da Vinci. Eur Heart J. 2014; $35: 1344$.

6. Katayama S, Umetani N, Sugiura S, Hisada T. The sinus of Valsalva relieves abnormal stress on aortic valve leaflets by facilitating smooth closure. J Thorac Cardiovasc Surg. 2008;136:1528-35.

7. Grande-Allen KJ, Cochran RP, Reinhall PG, Kunzelman KS. Re-creation of sinuses is important for sparing the aortic valve: a finite element study. $J$ Thorac Cardiovasc Surg. 2000;119:753-63.

8. Pisani G, Scaffa R, Ieropoli O, Dell'Amico EM, Maselli D, Morbiducci U, et al. Role of the sinuses of Valsalva on the opening of the aortic valve. J Thorac Cardiovasc Surg. 2013;145:999-1003.

9. Bellhouse BJ, Bellhouse FH. Mechanism of closure of the aortic valve. Nature. 1968:217:86-7.

10. Bellhouse BJ, Bellhouse FH, Reid KG. Fluid mechanics of the aortic root with application to coronary flow. Nature. 1968;219:1059-61.

11. Erasmi A, Sievers HH, Scharfschwerdt M, Eckel T, Misfeld M. In vitro hydrodynamics, cusp-bending deformation, and root distensibility for different types of aortic valve-sparing operations: remodeling, sinus prosthesis, and reimplantation. J Thorac Cardiovasc Surg. 2005;130:1044-9.

12. Fries R, Graeter T, Aicher D, Reul H, Schmitz C, Bohm M, et al. In vitro comparison of aortic valve movement after valve-preserving aortic replacement. J Thorac Cardiovasc Surg. 2006;132:32-7.

13. Kallenbach K, Kojic D, Oezsoez M, Bruckner T, Sandrio S, Arif R, et al. Treatment of ascending aortic aneurysms using different surgical techniques: a single-centre experience with 548 patients. Eur J Cardiothorac Surg. 2013;44:337-45.

14. David TE, Feindel CM, David CM, Manlhiot C. A quarter of a century of experience with aortic valve-sparing operations. J Thorac Cardiovasc Surg. 2014;148: 872-80.
15. Schmidtke C, Sievers HH, Frydrychowicz A, Petersen M, Scharfschwerdt M, Karluss A, et al. First clinical results with the new sinus prosthesis used for valve-sparing aortic root replacement. Eur J Cardiothorac Surg. 2013;43: 585-90.

16. Richardt D, Karluss A, Schmidtke C, Sievers HH, Scharfschwerdt M. A new sinus prosthesis for aortic valve-sparing surgery maintaining the shape of the root at systemic pressure. Ann Thorac Surg. 2010;89:943-6.

17. Kilner PJ, Yang GZ, Mohiaddin RH, Firmin DN, Longmore DB. Helical and retrograde secondary flow patterns in the aortic arch studied by three-directional magnetic resonance velocity mapping. Circulation. 1993;88:2235-47.

18. Bock J, Frydrychowicz A, Lorenz R, Hirtler D, Barker AJ, Johnson KM, et al In vivo noninvasive 4D pressure difference mapping in the human aorta: phantom comparison and application in healthy volunteers and patients. Magn Reson Med. 2011;66:1079-88.

19. Hennemuth A, Friman O, Schumann C, Bock J, Drexl J, Huellebrand M, et al Fast interactive exploration of 4D MRI flow data. Proc SPIE 7964, Medical Imaging 2011: Visualization, Image-Guided Procedures, and Modeling. 2011:79640E.

20. Buonocore MH. Visualizing blood flow patterns using streamlines, arrows, and particle paths. Magn Reson Med. 1998;40:210-26.

21. Markl M, Frydrychowicz A, Kozerke S, Hope M, Wieben O. 4D flow MRI J Magn Reson Imaging. 2012;36:1015-36.

22. Dyverfeldt P, Bissell M, Barker AJ, Bolger AF, Carlhall CJ, Ebbers T, et al. 4D flow cardiovascular magnetic resonance consensus statement. J Cardiovasc Magn Reson. 2015;17:72.

23. Frydrychowicz A, Berger A, Munoz Del Rio A, Russe MF, Bock J, Harloff A et al. Interdependencies of aortic arch secondary flow patterns, geometry, and age analysed by 4-dimensional phase contrast magnetic resonance imaging at 3 Tesla. Eur Radiol. 2012;22:1122-30.

24. Kvitting JP, Ebbers T, Wigstrom L, Engvall J, Olin CL, Bolger AF. Flow pattern in the aortic root and the aorta studied with time-resolved, 3-dimensional, phasecontrast magnetic resonance imaging: implications for aortic valve-sparing surgery. J Thorac Cardiovasc Surg. 2004;127:1602-7.

25. Markl M, Draney MT, Miller DC, Levin JM, Williamson EE, Pelc NJ, et al. Time-resolved three-dimensional magnetic resonance velocity mapping of aortic flow in healthy volunteers and patients after valve-sparing aortic root replacement. J Thorac Cardiovasc Surg. 2005;130:456-63.

26. Frydrychowicz A, Landgraf B, Markl M. Controversial findings in "three-dimensional velocity mapping of thoracic aorta and supra-aortic arteries in Takayasu arteritis." J Magn Reson Imaging. 2011;33:252-3.

27. Van Steenhoven AA, Van Dongen MEH. Model studies of the closing behaviour of the aortic valve. J Fluid Mechanics. 1979;90:21-32.

28. Spencer MP, Greiss FC. Dynamics of ventricular ejection. Circ Res. 1962;10 274-9.

29. Driscol TE, Eckstein RW. Systolic pressure gradients across the aortic valve and in the ascending aorta. Am J Physiol. 1965;209:557-63.

30. Caro CG, Pedley TJ, Schroter RC, Seed WA. The Mechanics of the Circulation 2nd ed. Cambridge: Cambridge University Press; 2012:229-31.

31. Tyszka JM, Laidlaw DH, Asa JW, Silverman JM. Three-dimensional, timeresolved (4D) relative pressure mapping using magnetic resonance imaging. J Magn Reson Imaging. 2000;12:321-9.

32. Lum DP, Johnson KM, Paul RK, Turk AS, Consigny DW, Grinde JR, et al. Trans stenotic pressure gradients: measurement in swine-retrospectively ECG-gated 3D phase-contrast MR angiography versus endovascular pressure-sensing guidewires. Radiology. 2007;245:751-60.

33. De Paulis R, Chirichilli I, Scaffa R, Weltert L, Maselli D, Salica A, et al. Longterm results of the valve reimplantation technique using a graft with sinuses. J Thoracic Cardiovasc Surg. 2016;151:112-9.

34. Jung B, Honal M, Ullmann P, Hennig J, Markl M. Highly k-t-space-accelerated phase-contrast MRI. Magn Reson Med. 2008;60:1169-77.

35. Gu T, Korosec FR, Block WF, Fain SB, Turk Q, Lum D, et al. PC VIPR: a highspeed 3D phase-contrast method for flow quantification and high-resolution angiography. Am J Neuroradiol. 2005;26:743-9.

Key Words: sinus prosthesis, 4D Flow MRI, 4D flow cardiovascular magnetic resonance, sinus vortices, sinuses of Valsalva, transvalvular pressure gradients, valve-sparing aortic root replacement, VSARR 


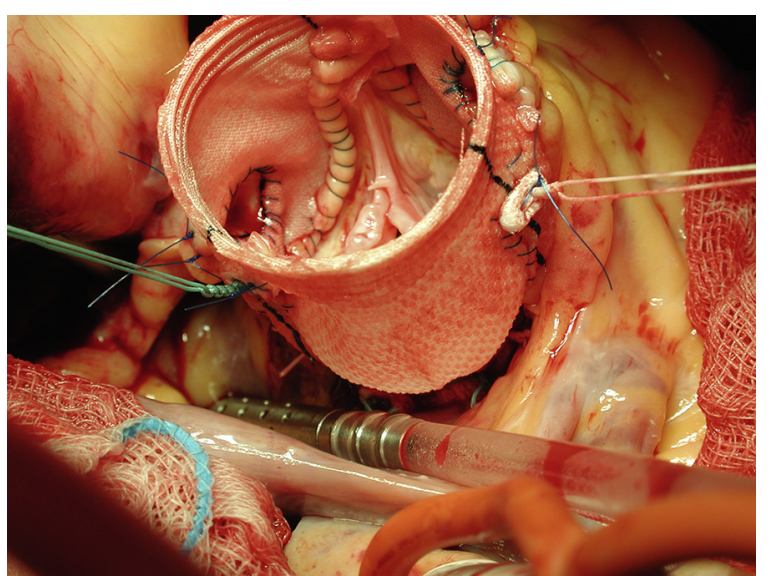

FIGURE E1. The sinus prosthesis. Intraoperative view of an implanted sinus prosthesis. Note the sinus configuration and the straight pillars between the sinuses. 